ARTICLE

\title{
Regulation of cardiomyocyte behavior in zebrafish trabeculation by Neuregulin 2a signaling
}

S. Javad Rasouli ${ }^{1} \&$ Didier Y.R. Stainier ${ }^{1}$

Trabeculation is crucial for cardiac muscle growth in vertebrates. This process requires the Erbb2/4 ligand Neuregulin ( $\mathrm{Nrg}$ ), secreted by the endocardium, as well as blood flow/cardiac contractility. Here, we address two fundamental, yet unresolved, questions about cardiac trabeculation: why does it initially occur in the ventricle and not the atrium, and how is it modulated by blood flow/contractility. Using loss-of-function approaches, we first show that zebrafish Nrg2a is required for trabeculation, and using a protein-trap line, find that it is expressed in both cardiac chambers albeit with different spatiotemporal patterns. Through gain-of-function experiments, we show that atrial cardiomyocytes can also respond to Nrg2a signalling, suggesting that the cardiac jelly, which remains prominent in the atrium, represents a barrier to Erbb2/4 activation. Furthermore, we find that blood flow/contractility is required for Nrg2a expression, and that while non-contractile hearts fail to trabeculate, non-contractile cardiomyocytes are also competent to respond to Nrg2a/Erbb2 signalling.

\footnotetext{
${ }^{1}$ Department of Developmental Genetics, Max Planck Institute for Heart and Lung Research, 61231 Bad Nauheim, Germany. Correspondence and requests for materials should be addressed to D.Y.R.S. (email: Didier.Stainier@mpi-bn.mpg.de).
} 
C ardiac trabeculation, which allows the increase in muscle mass prior to the formation of coronaries, is one of the most important processes the vertebrate heart must undergo to form and function properly ${ }^{1}$. During this process, trabeculae, which are sponge-like muscular structures in the cardiac cavity, form as a result of cardiomyocyte delamination and proliferation ${ }^{2-6}$. Defects in this complex morphogenetic event lead to embryonic lethality or adult onset dilated cardiomyopathy ${ }^{5,7-12}$ illustrating the importance of trabeculation in cardiac development and function.

The vertebrate heart initially consists of two cell layers, the endocardium and myocardium, separated by an acellular, gelatinous matrix called the cardiac jelly ${ }^{13-15}$. Ablating components of the cardiac jelly by injecting hyaluronidase in chick embryos has been reported to cause looping defects ${ }^{16}$, indicating that it plays an important role during early cardiac morphogenesis. Endocardial-myocardial interactions are known to orchestrate cardiac trabeculation; for example, in chick embryos, a myocardial-derived growth factor, Angiopoietin 1, has been reported to bind to its receptor Tie 2 which is expressed by endocardial cells and modify their angiogenic behaviour which in turn affects the complexity of cardiac trabeculation ${ }^{17}$. Similarly, signalling from endocardial to myocardial cells is also necessary for trabeculation. For instance, mice lacking the endocardial-derived ligand Nrg1 or its receptors Erbb2 and Erbb4, which are expressed by myocardial cells, exhibit severe trabeculation defects ${ }^{7,8,12,18}$. Similarly, zebrafish erbb2 mutants fail to form trabeculae ${ }^{5}$. Additional studies have shown that Nrg signalling can positively regulate cardiomyocyte proliferation and heart regeneration ${ }^{19-22}$.

Cardiac formation can also be influenced by its function ${ }^{23-25}$. Recent studies have shown the dependence of trabeculation on physical forces generated by cardiac contractility and blood flow $6,26,27$. For instance, several groups have reported that reduction of blood flow in the ventricular chamber markedly diminishes trabeculation in chick embryos and zebrafish larvae $6,26,28,29$. By manipulating cardiac contractility, another study presented data indicating that cardiac contraction promotes trabeculation through the regulation of Notch signalling in the ventricular endocardium ${ }^{27}$. These and other findings support the hypothesis that physical forces are necessary in the developing heart, particularly during trabecular formation.

In this study, we use the zebrafish model to address two fundamental questions pertinent to cardiac trabeculation; first, why trabeculae initially form in the ventricle and not the atrium, as is observed in zebrafish as well as in other vertebrates including amniotes $^{3,30}$, and second, how trabeculation is modulated by physical forces. Using a genetic approach and high-resolution live imaging, we first show that Nrg2a is an essential ligand for cardiac trabeculation in zebrafish. By ectopically expressing $n r g 2 a$ in cardiomyocytes, we further show that atrial cardiomyocytes, like ventricular cardiomyocytes, can respond to Nrg2a/Erbb2 signalling. Using loss- and gain-of-function approaches, we show that blood flow/contractility modulates endocardial $n r g 2 a$ expression while it is not required for the expression or function of $\mathrm{Nrg} 2 \mathrm{a} / \mathrm{Erbb} 2$ signalling effectors in cardiomyocytes.

\section{Results}

Nrg2a is required for cardiac trabeculation in zebrafish. Given the role of Nrg1 in cardiac trabeculation in mouse $e^{7,8,12,18}$, we mutated zebrafish $n r g 1$ using TALEN technology ${ }^{31}$. Since $n r g 1$ has multiple isoforms ${ }^{32}$, we targeted exon 2 which encodes part of the highly conserved IGc2-domain, and identified a $\Delta 14$ allele (Supplementary Fig. 1a) encoding a predicted truncated protein (Supplementary Fig. 1b) due to harbouring a premature stop codon within the same exon. Using brightfield microscopy, no gross morphological defects were observed in $n r g 1 \Delta 14$ ( $\left.\operatorname{rrgl}^{\text {bns101}}\right)$ homozygous mutant larvae (Supplementary Fig. 1c,d). Incrossing $n r g 1^{b n s 101 /+}$ fish in the Tg(myl7:LIFEACT-GFP) background, a myocardial specific line in which F-actin (filamentous actin) is labelled with $\mathrm{GFP}^{33}$, we observed that trabeculation appears unaffected in $n r g 1^{\text {bns } 101}$ mutant larvae (Supplementary Fig. 1e-h), which can survive to become fertile adults.

Next, we decided to investigate the role of Nrg2a, another member of the Nrg family, by using a conditional protein-trap line, $n r g 2 a^{m n 0237 G t}$ (refs 34,35). Using brightfield microscopy, $n r g 2 a$ mutants can be recognized by their aberrant jaws (Fig. 1a,b) as well as other defects in median fin fold (MFF) morphogenesis and swim bladder inflation ${ }^{35}$. As previously reported, the gene-breaking transgene (GBT) $n r g 2 a$ allele generates a truncated Nrg2a protein tagged with mRFP (Nrg2a-mRFP) due to the in-frame integration of the mutagenic pGBT-RP2.1 (RP2) cassette in the intron between alternative exon $1 \mathrm{C}$ and exon 2 (ref. 35). Prior studies have also shown that the expression of Nrg2a-mRFP recapitulates that of endogenous $n r g 2 a$ (refs 34,35). Confocal imaging of larvae from $n r g 2 a^{+/-}$ incrosses in the $\operatorname{Tg}(m y l 7: L I F E A C T-G F P)$ background (Fig. 1c-k) revealed that Nrg2a-mRFP is highly expressed in the endocardium at early larval stages (Fig. 1d,e,g,h,j,k), and that $n r g 2 a^{-1-}$ hearts fail to form trabeculae by $75 \mathrm{~h}$ post fertilization (hpf) (Fig. 1c-e). Observation of $n r g 2 a$ mutants at later stages, including 120 and $168 \mathrm{hpf}$ (Fig. 1f-k), confirmed that their trabeculation defects appear as severe as those in erbb2 mutants ${ }^{5}$. Overall, these data suggest that Nrg2a, and not Nrg1, is the crucial regulator of cardiac trabeculation in zebrafish.

Differential Nrg2a expression in the ventricle and atrium. Trabeculae start to form in zebrafish around $60 \mathrm{hpf}$ and become clearly apparent by $72 \mathrm{hpf}^{5,26}$. To investigate why trabeculation occurs primarily in the ventricle and not the atrium ${ }^{3,30}$, we examined Nrg2a-mRFP expression at several stages of cardiac development. We used high-resolution confocal microscopy to image live $n r g 2 a^{+/-}$animals in the $T g(k d r l: N L S-E G F P)$ background, in which the endothelial cell nuclei are labelled with $\mathrm{GFP}^{36}$, at 52, 78 and $120 \mathrm{hpf}$ (Fig. 2a-i). Nrg2a-mRFP expression appears in the endocardium at $52 \mathrm{hpf}$, particularly in the outer curvature of the ventricle (Fig. 2a-c, Supplementary Fig. $2 \mathrm{a}-\mathrm{c}$ and Fig. 3a-c). Subsequently, Nrg2a-mRFP expression becomes stronger in ventricular and atrial endocardial cells (Fig. 2d-i, Supplementary Fig. 2d-i, Fig. 3d-i), although it is weaker in the atrioventricular (AV) canal (Fig. 2d-i and Supplementary Fig. 4a-c). Accordingly, $n r g 2 a$ mutants develop functional AV valve leaflets (Supplementary Fig. $4 \mathrm{~d}-\mathrm{i}$ ) as was observed in erbb2 mutants $^{5}$, suggesting that Nrg2a/Erbb2 signalling is not required for $\mathrm{AV}$ valve development.

Utilizing the Zeiss Efficient Navigation (ZEN) software, we measured the levels of Nrg2a-mRFP expression as well as the number of Nrg2a-mRFP positive endocardial cells at $78 \mathrm{hpf}$ (Fig. 2j,k). These analyses show that ventricular endocardial cells express higher levels of Nrg2a-mRFP than atrial or AV canal endocardial cells (Fig. 2j), and that there are more Nrg2a-mRFP positive endocardial cells in the ventricle as compared to the other cardiac regions (Fig. 2k). We also found that on average $(N=5$ hearts), $97 \%$ and $90 \%$ of endocardial cells in the ventricle and atrium were positive for Nrg2a-mRFP expression, respectively (Supplementary Fig. 2j). Thus, ventricular cardiomyocytes might be exposed to a higher level of Nrg2a ligands than atrial ones, although atrial endocardial cells are clearly positive for Nrg2a-mRFP expression. 

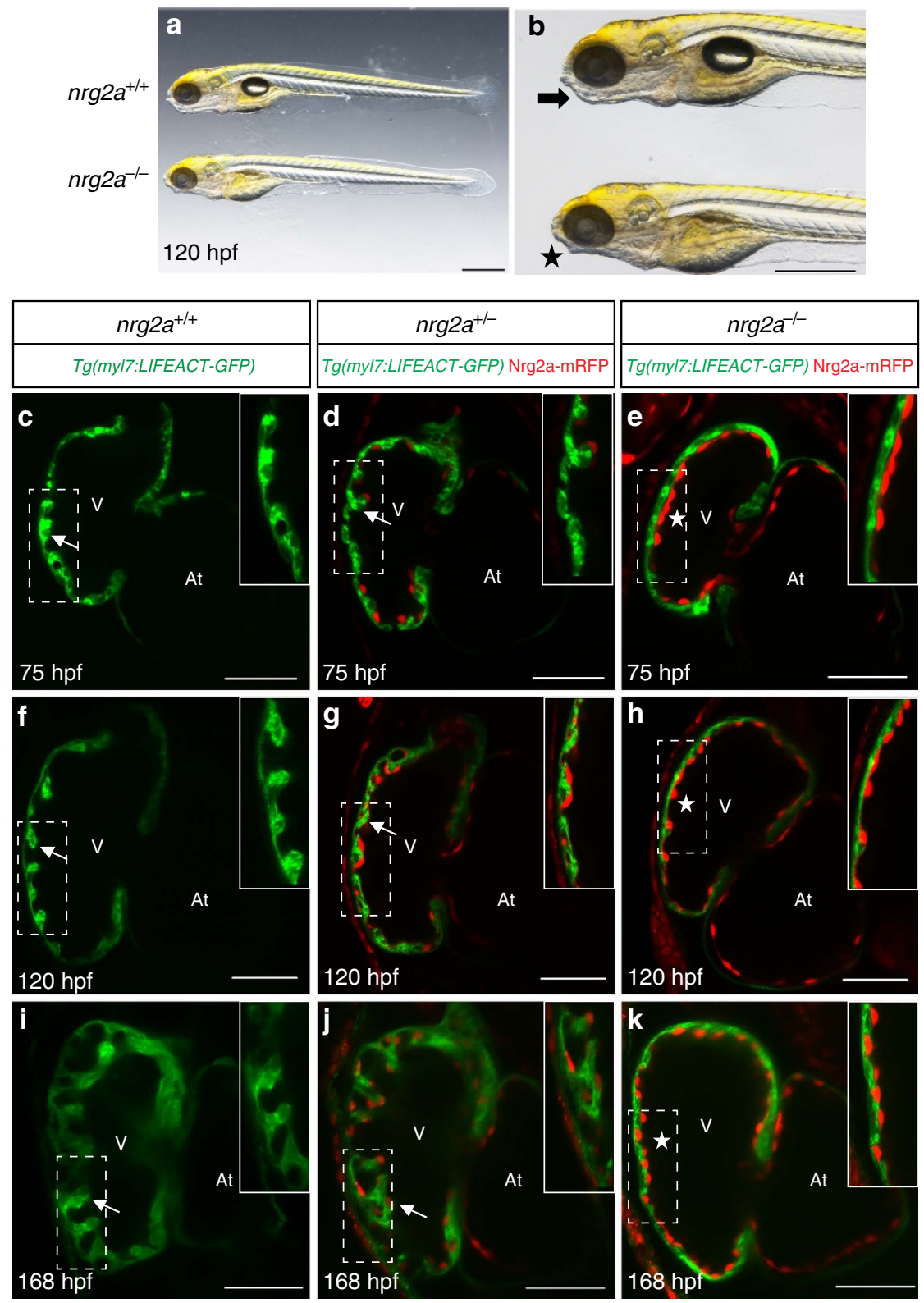

Figure 1 | Nrg2a is required for cardiac trabeculation in zebrafish. (a,b) Zebrafish larvae from nrg $2 a^{+/-}$incrosses were imaged at 120 hpf; lateral view, anterior to the left; scale bars, $0.5 \mathrm{~mm}$. $n r g 2 a^{-/-}$larvae can be recognized by their defective jaws (asterisk in b). (c-k) nrg2a mutants lack cardiac trabeculae. Confocal images (mid-sagittal sections) of larval hearts from Tg(myl7:LIFEACT-GFP);nrg2a+/- incrosses at 75 (c-e), 120 (f-h) and 168 hpf (i-k); ventricular outer curvature (dashed boxes) magnified in upper right corners; arrows and asterisks indicate trabeculated and non-trabeculated walls, respectively; scale bars, $50 \mu \mathrm{m}$.

To examine whether the Nrg2a protein-trap expression in the endocardium accurately recapitulates endogenous $n r g 2 a$ expression $^{34,35}$, we carried out in situ hybridizations on $78 \mathrm{hpf}$ hearts. We observed that $n r g 2 a$ is more highly expressed in the ventricle, compared to the atrium, and that it is mainly restricted to the outer curvature (Supplementary Fig. 2k). This expression pattern is consistent with that of Nrg2a-mRFP.

The cardiac jelly and the onset of trabeculation. The cardiac jelly is known to be pivotal in endo-myocardial interactions required for cardiac morphogenesis 4,37 , and its reduction during development has previously been reported in chicken and mice ${ }^{4,38}$. Using high-resolution confocal microscopy, we decided to investigate more closely the dynamics of cardiac jelly reduction around the onset of trabeculation in $\mathrm{Tg}(\mathrm{kdrl}: H s a . H R A S-m C h e r r y)^{39} ; \mathrm{Tg}(m y l 7: E G F P-H s a . H R A S)^{40}$ animals in which endocardial and myocardial membranes are labelled in red and green, respectively. We found that at $48 \mathrm{hpf}$ the cardiac jelly fully separates the endocardial and myocardial layers (Fig. 3a), and that its thickness gradually decreases in both chambers but more clearly in the ventricle (Fig. $3 b-h$ ). The timing of this decrease correlates with the appearance of the first trabeculae in the outer curvature of the ventricle by $60 \mathrm{hpf}$ (Fig. 3b,c, arrowhead). By $96 \mathrm{hpf}$, the cardiac jelly appears to be fully gone in the ventricle while it remains in the atrium for at least the first 6 days of development (Fig. 3d-h).

To investigate whether $\mathrm{Nrg} 2 \mathrm{a}$ signalling modulates the thickness of the cardiac jelly, we incrossed $n r g 2 a^{+/-}$animals in the $T g(k d r l: H s a . H R A S-m C h e r r y) ; T g(m y l 7: L I F E A C T-G F P)$ 
background, and found no obvious differences between wild types and mutants (Supplementary Fig. 5a-h), similar to what we observed in $n r g 1^{\text {bns101 }}$ mutants and Erbb2 inhibitor-treated animals (Supplementary Fig. 5i-k), suggesting that Nrg/Erbb2 signalling is not involved in this process in zebrafish.

Altogether, these data show that the reduction of the cardiac jelly takes place in the ventricle as trabeculation starts, suggesting that it may determine the onset of this process, possibly by constituting a diffusion barrier for Nrg ligands.

nrg2a overexpression in cardiomyocytes. The current model is that endocardial-derived Nrg ligands traverse the cardiac jelly to activate cardiomyocyte behaviour and proliferation ${ }^{4,12,41,42}$. To
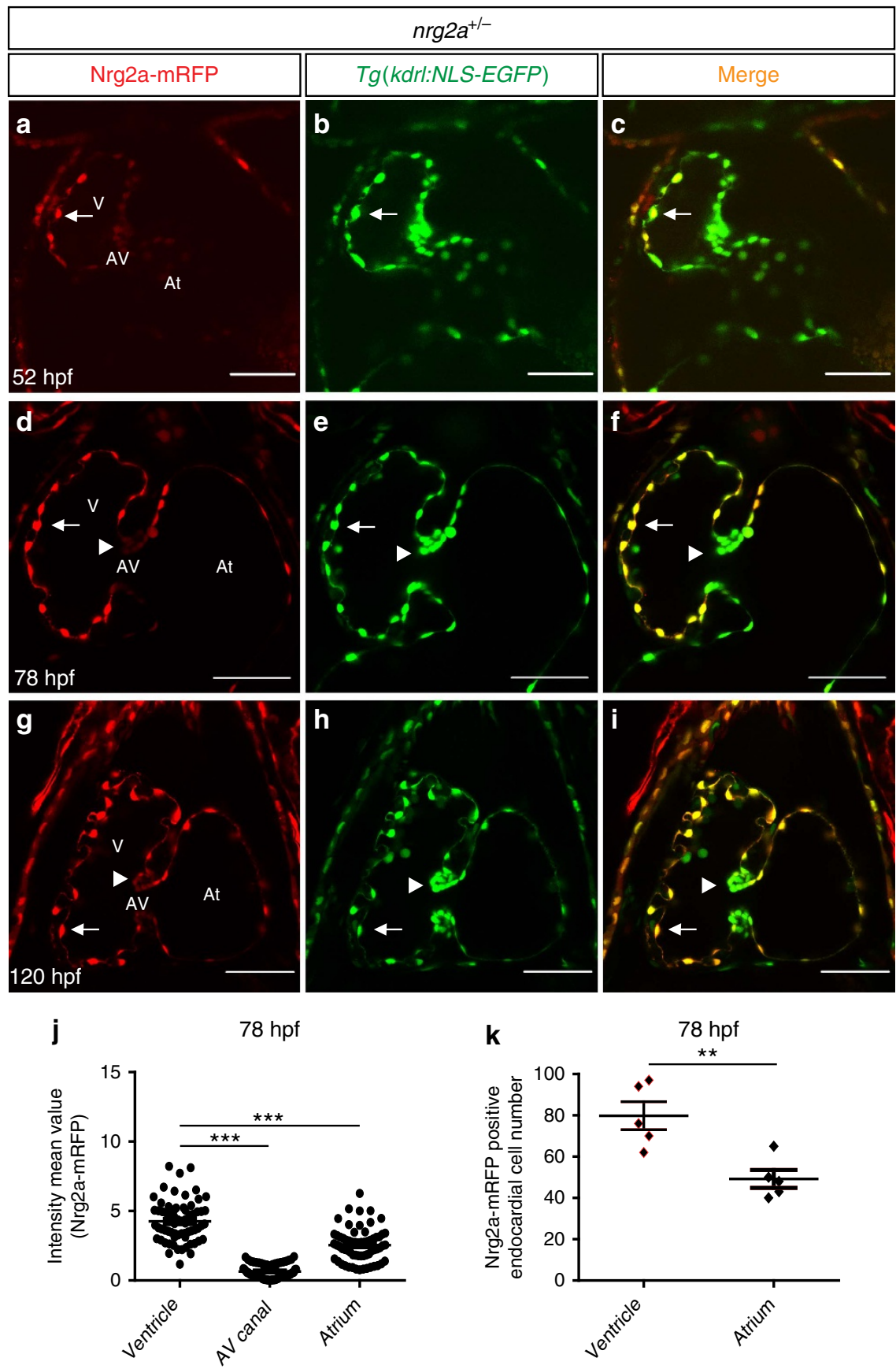

Figure 2 | Nrg2a-mRFP expression during embryonic and larval cardiac development. (a-i) Two-dimensional (2D) confocal images (mid-sagittal sections) of zebrafish hearts from $\mathrm{Tg}(\mathrm{kdrl}: \mathrm{NLS}-\mathrm{EGFP}) ; n r g 2 a^{+/-}$outcrosses at 52 (a-c), 78 (d-f) and 120 hpf (g-i) showing that Nrg2a-mRFP expression is clearly visible in the ventricular endocardium by $52 \mathrm{hpf}$, mainly in the outer curvature (a-c), and that it becomes stronger in both ventricular and atrial chambers at 78 and $120 \mathrm{hpf}(\mathbf{d}-\mathbf{i})$; however, it is weak in the atrioventricular canal; arrowheads point to the superior valve leaflet (a-i); AV, atrioventricular canal, At, atrium; V, ventricle; scale bars, $50 \mu \mathrm{m}$. (j) Cell-based mRFP intensity, measured with the ZEN Imaging Software and plotted as a graph, showing that Nrg2a-mRFP is more highly expressed in the ventricle compared to the AV canal and atrium; dots in this graph represent individual Nrg2a-mRFP expressing endocardial cells. (k) Nrg2a-mRFP positive endocardial cells counted in each chamber at 78 hpf, showing that there are more Nrg2a-mRFP positive endocardial cells in the ventricle than in the atrium at $78 \mathrm{hpf}$; dots in this graph represent individual hearts; $N=5$ hearts; values represent means \pm s.e.m.; ${ }^{\star \star} P \leq 0.01,{ }^{\star \star \star} P \leq 0.001$ by Student's $t$-test. 

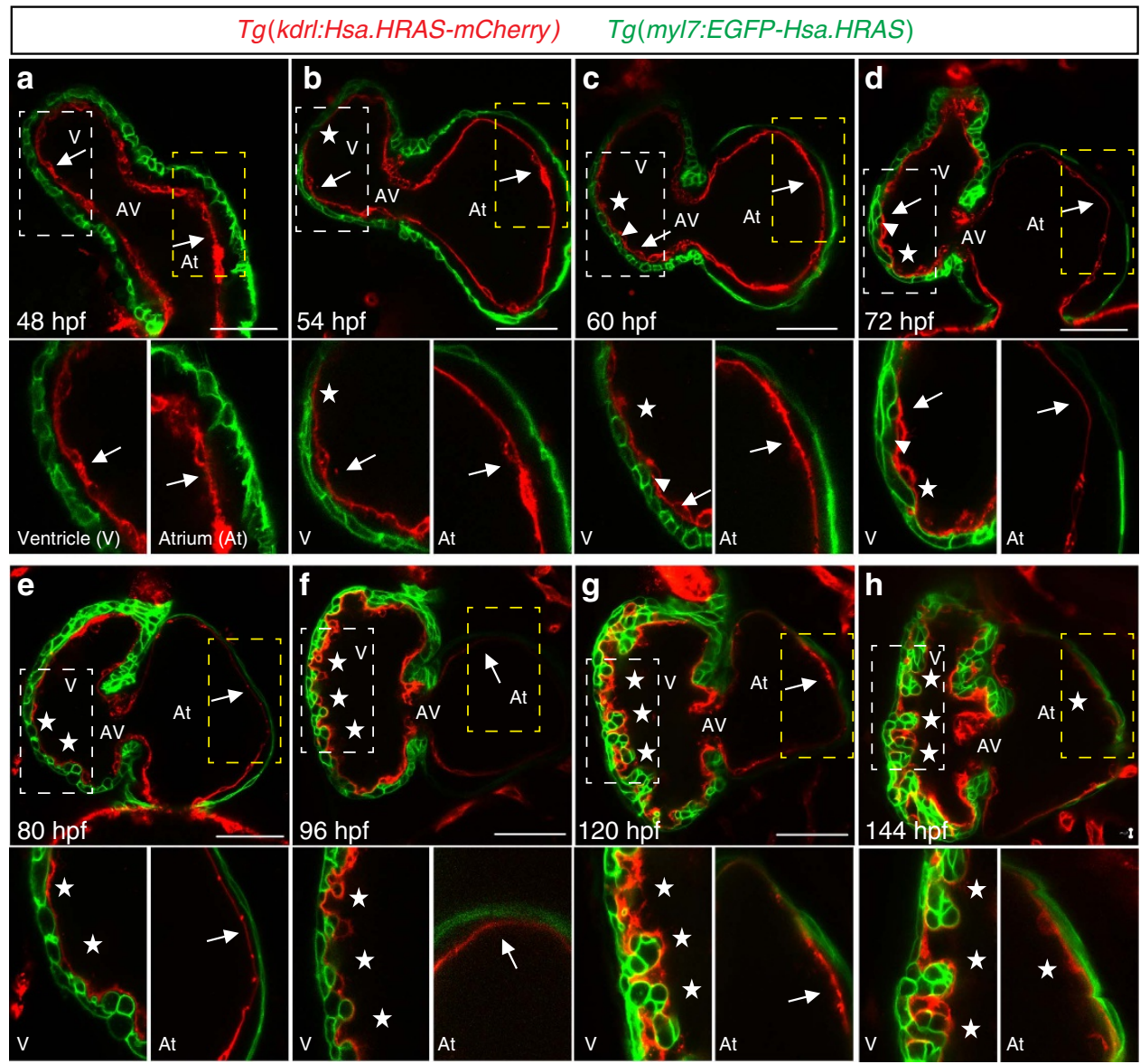

Figure 3 | Reduction of cardiac jelly thickness in developing zebrafish. (a-h) Mid-sagittal confocal sections of late embryonic and early larval zebrafish hearts. Animals from $\mathrm{Tg}(\mathrm{kdrl}: H s a . H R A S-m C h e r r y) ; \mathrm{Tg}(m y l 7: E G F P-H s a . H R A S)$ incrosses were synchronized at the tail bud stage (10 hpf) and imaged at 48 (a), 54 (b), 60 (c), 72 (d), 80 (e), 96 (f), 120 (g) and 144 hpf (h); endocardial and myocardial membranes are labelled in red and green, respectively; AV, atrioventricular canal, At, atrium; V, ventricle; scale bars, $50 \mu \mathrm{m}$. Higher magnification images of outer curvature area of ventricular (white dashed boxes) and atrial (yellow dashed boxes) walls are shown beneath each time point, respectively. The cardiac jelly is initially thicker in the atrium than in the ventricle at $48 \mathrm{hpf}$ (a). It is greatly reduced in the ventricle by $72 \mathrm{hpf},(\mathbf{b}-\mathbf{d})$ and almost fully gone by $96 \mathrm{hpf}$, while it is still detectable at $120 \mathrm{hpf}$ in the atrium (e-h); arrowheads point to trabecular cardiomyocytes; arrows and asterisks indicate the presence and absence of cardiac jelly, respectively.

determine whether ectopic overexpression of $n r g 2 a$ in cardiomyocytes enables them to form trabeculae in $n r g 2 a$ mutants, we collected embryos from $\mathrm{Tg}(\mathrm{myl}$ : LIFEACTGFP); $n r g 2 a^{+/}$incrosses and injected them with a $m y l 7: n r g 2 a-$ p2a-tdTomato plasmid at the one-cell stage (Fig. 4a,b). This approach allows one to identify the $n r g 2 a$ overexpressing cardiomyocytes by virtue of their expression of tdTomato. Confocal microscopy analysis showed that ectopic overexpression of $n r g 2 a$ in $n r g 2 a$ mutant ventricular cardiomyocytes could drive them to form a multilayered wall in most tdTomato positive hearts $(13<15)$ (Fig. $4 \mathrm{c}-\mathrm{e})$. To determine whether Nrg2a signals via Erbb2 in cardiomyocytes, we ectopically overexpressed $n r g 2 a$ in erbb2 $2^{\text {st } 61}$ mutant cardiomyocytes. Our results show that mosaic myocardial $n r g 2 a$ overexpression did not rescue the trabeculation defects in erbb2 mutants (Fig. $4 \mathrm{f}-\mathrm{i}$ ), indicating that Nrg2a must signal through Erbb2 in cardiomyocytes.

We also generated a stable myocardial-specific Nrg2a transgenic line by injecting wild-type embryos with the myl7:nrg2a-p2a-tdTomato plasmid. Although a majority of the injected animals survived to adulthood, some exhibited pericardial edema due to the presence of a much enlarged heart as compared to those of non-injected animals, indicating that mosaic expression of $n r g 2 a$ in cardiomyocytes leads to cardiomegaly (Supplementary Fig. 6a-d), as expected from data in mouse using a transgene encoding constitutively active Erbb2 (ref. 21) and in zebrafish using $n r g 1$ overexpression ${ }^{22}$. Overall, these data indicate that ventricular cardiomyocytes are capable to respond to the myocardial overexpression of $n r g 2 a$.

Atrial cardiomyocytes can respond to Nrg2a signalling. To further investigate the correlation between cardiac jelly thickness and the onset of trabeculation, we used our newly generated $T g$ (myl7:nrg2a-p2a-tdTomato) line to evaluate the behaviour of cardiomyocytes in the presence or absence of Nrg2a signalling (Fig. 5a). The cardiac jelly is present for at least the first 6 days of development in the zebrafish atrium, physically separating the endocardial and myocardial walls (Fig. 3), and thus possibly preventing myocardial cells from receiving endocardial-derived Nrg2a molecules. A myocardial specific $n r g 2 a$ overexpression approach allows one to circumvent the hypothetical barrier function of the cardiac jelly and test whether atrial cardiomyocytes are in fact competent to respond to $\mathrm{Nrg}$ signalling. To better visualize cardiomyocyte boundaries, Tg(myl7:nrg2a-p2a-tdTomato) fish were outcrossed to the myocardial specific membrane line $\mathrm{Tg}(\mathrm{myl7}$ :EGFP-Hsa.HRAS). Compared to $\mathrm{Tg}$ (myl7:nrg2a-p2a-tdTomato) negative animals, we observed that $n r g 2 a$ overexpression in cardiomyocytes pushed 
a

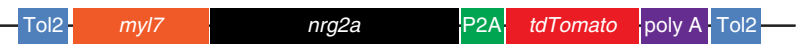

\section{b}

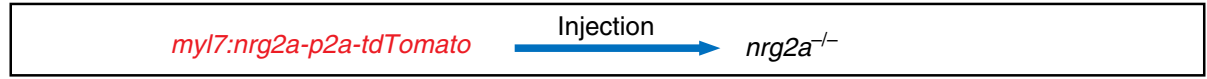

myl7:nrg2a-p2a-tdTomato
$\operatorname{Tg}($ myl7:LIFEACT-GFP) Nrg2a-mRFP $\quad \operatorname{Tg}$ (myl7:LIFEACT-GFP) $\begin{gathered}\text { myl7:nrg2a-p2a-tdTomato } \\ \text { Nrg2a-mRFP }\end{gathered}$
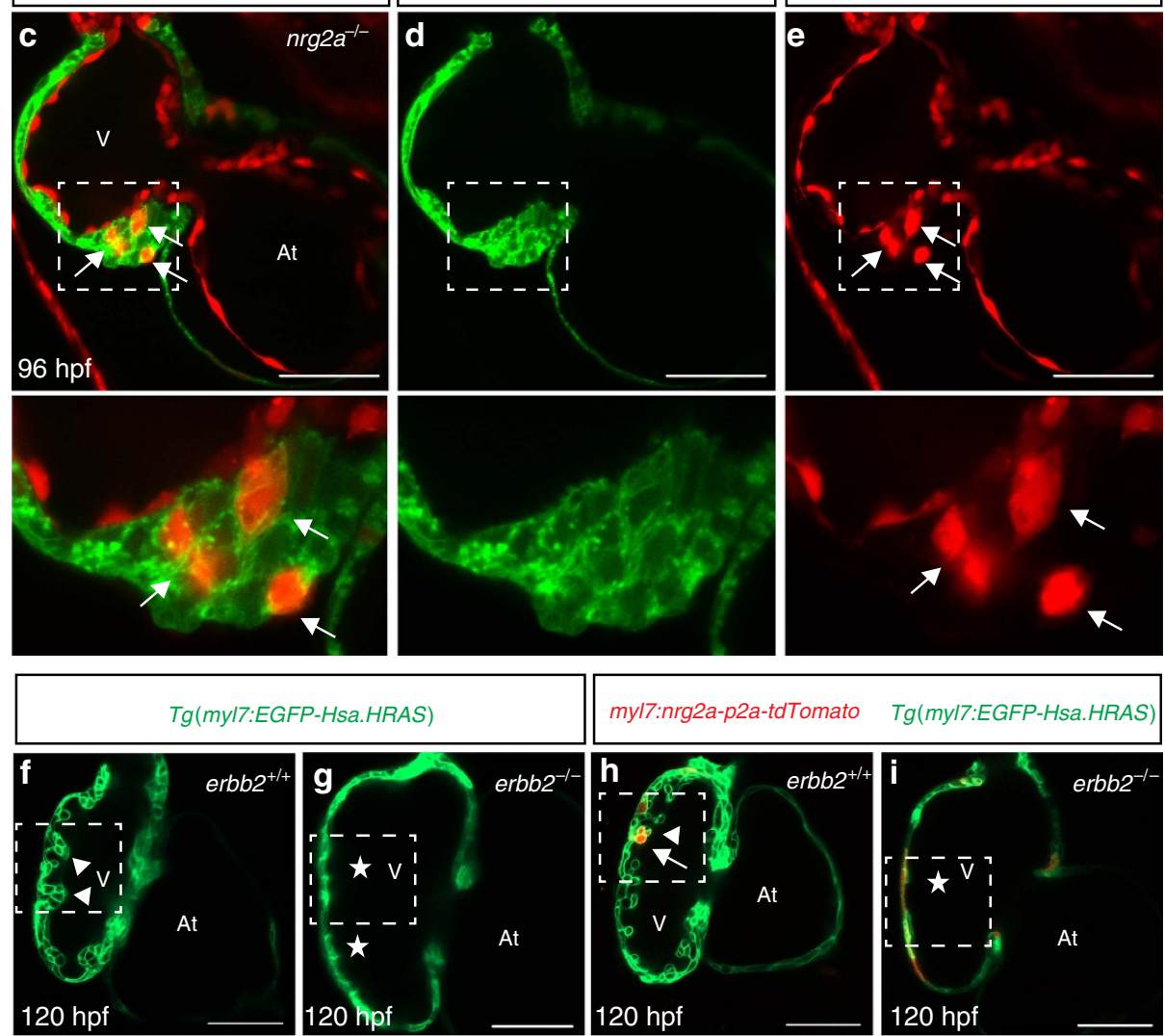

P-Hsa.HRAS myl7:nrg2a-p2a-tdTomato $\operatorname{Tg}($ myl7:EGFP-Hsa.HRAS)
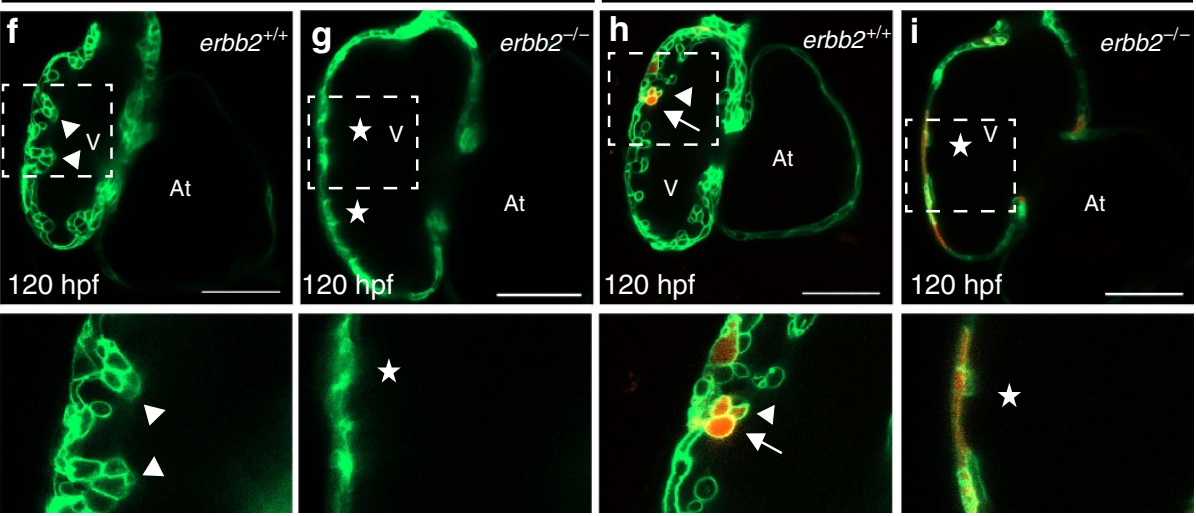

Figure 4 | Myocardial nrg2a overexpression induces cardiomyocyte multilayering in nrg2a mutants. (a) Cartoon of myocardial specific nrg2a construct. (b) Schematic representation of Nrg2a protein tagged by tdTomato. Due to the presence of the P2A peptide, cleavage occurs right after protein translation to separate the Nrg2a from the tdTomato fluorescent protein. (c-e) 2D confocal images (mid-sagittal sections) of $\mathrm{Tg}$ (myl7:LIFEACT-GFP);nrg2a $-/-$ hearts injected with myocardial specific nrg2a construct (myl7:nrg2a-p2a-tdTomato) at the one-cell stage. Mosaic overexpression of $n r g 2 a$ in $n r g 2 a-/-$

cardiomyocytes led to the formation of a multilayered myocardial wall which is outlined by a white dashed box and magnified (c-e); arrows point to $n r g 2 a$ overexpressing cardiomyocytes. (f-i) Confocal images (mid-sagittal sections) of $120 \mathrm{hpf}$ Tg(myl7:EGFP-Hsa.HRAS) hearts from erbb2 $+/-$ incrosses injected with the myl7:nrg2a-p2a-tdTomato construct (h-i). Magnified images of dashed boxes are shown below $\mathbf{c}-\mathbf{i}$; arrows point to nrg2a overexpressing cardiomyocytes, arrowheads point to trabeculae and asterisks indicate lack of trabeculae. At, atrium; $\mathrm{V}$, ventricle; scale bars, $50 \mu \mathrm{m}$.

them to form a bilayered wall in the ventricle as well as the atrium starting at 46 and $78 \mathrm{hpf}$, respectively (Fig. 5b-e, Supplementary Fig. 7a,b). Subsequently, constitutively active Nrg2a signalling resulted in cardiomyocyte multilayering in both ventricular and atrial walls (Fig. 5f-i). However, this cardiomyocyte response was blocked by Erbb2 inhibitor treatment starting at $36 \mathrm{hpf}$ (Supplementary Fig. 8a-d). By crossing the $\operatorname{Tg}(m y l 7: n r g 2 a-p 2 a-$ tdTomato) line with a Fucci line (Tg(myl7:mVenus-gmnn)) which labels proliferating cardiomyocytes in green ${ }^{43}$, we observed that ectopic myocardial expression of $n r g 2 a$ increased the number of proliferating cardiomyocytes (Supplementary Fig. 9a-f). Overall, these data indicate that both ventricular and atrial cardiomyocytes can respond to Nrg/Erbb2 signalling.

Contractility regulates endocardial nrg2a expression. Physical forces generated by contractility have been previously shown to modulate cardiac trabeculation ${ }^{6,26,27}$. Taking advantage of the 

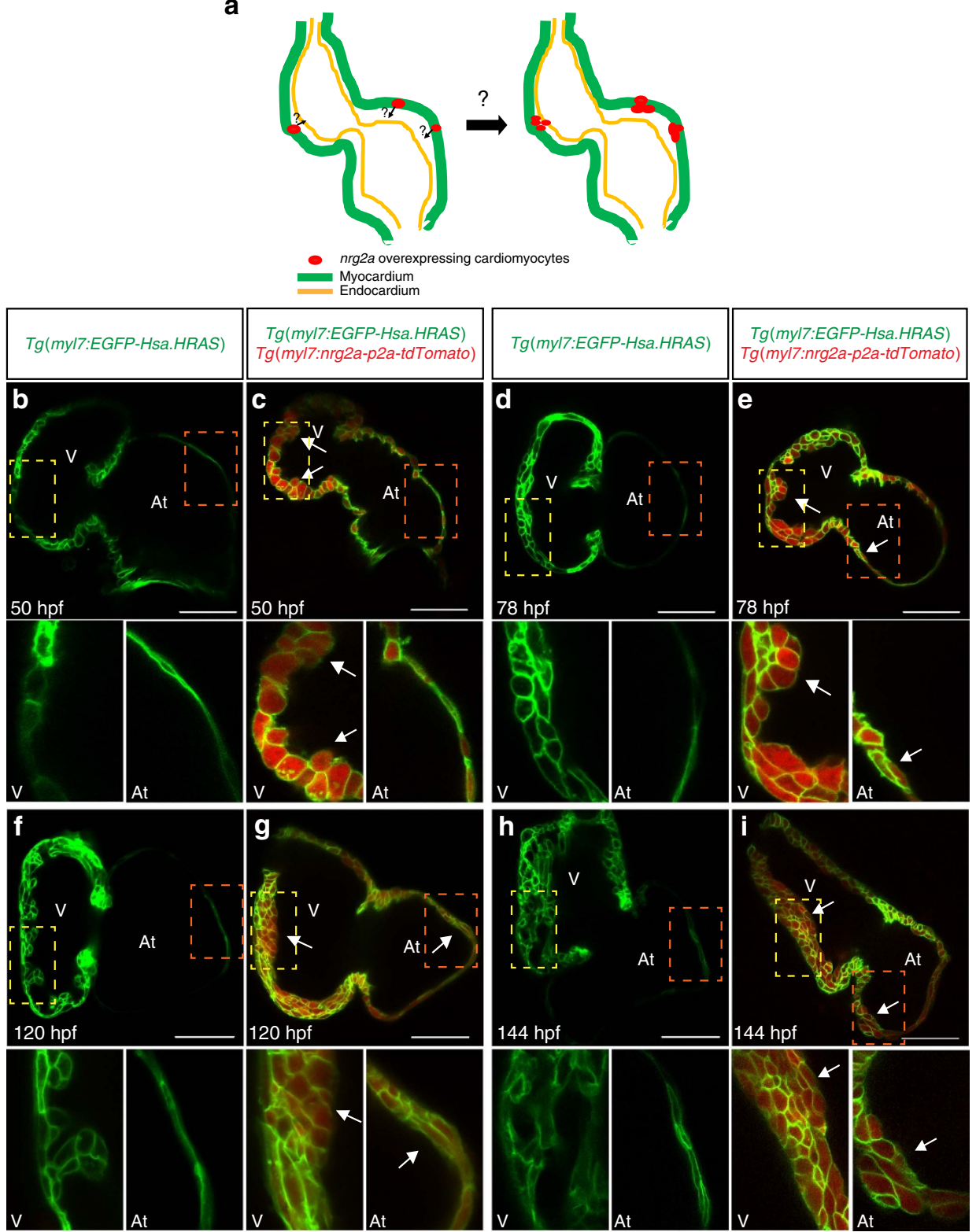

Figure 5 | Myocardial specific nrg2a overexpression can lead atrial cardiomyocytes to form a multilayered wall. (a) Illustration of cardiomyocyte specific nrg2a overexpression in zebrafish. (b-i) 2D confocal images (mid-sagittal views) of Tg(myl7:EGFP-Hsa.HRAS) and Tg(myl7:EGFP-

Hsa.HRAS); $\operatorname{Tg}($ myl7:nrg2a-p2a-tdTomato) hearts at $50(\mathbf{b}, \mathbf{c}), 78(\mathbf{d}, \mathbf{e}), 120(\mathbf{f}, \mathbf{g})$ and $144 \mathrm{hpf}(\mathbf{h}, \mathbf{i}) ;$ magnified images of dashed boxes are shown below each time point; arrows point to multilayered walls; At, atrium; $V$, ventricle; scale bars, $50 \mu \mathrm{m}$.

nrg2a protein-trap line, we wanted to investigate how cardiac contractility/blood flow regulates $n r g 2 a$ expression in the zebrafish heart. At $78 \mathrm{hpf}$, we observed that endocardial Nrg2amRFP is strongly expressed in the outer curvature of wild-type ventricles, where trabeculae are present, while it is clearly weaker in other endocardial cells (Fig. 6a-c,j). The levels of Nrg2a-mRFP expression in the heart were strongly reduced in the absence of cardiac contractility in tnnt $2 a$ morphants ${ }^{44}$ (Fig. 6d-f,k) and 2,3-butanedione monoxime (BDM)-treated larvae (Fig. 6g-i,l), indicating that contraction and/or blood flow is required for cardiac expression of Nrg2a-mRFP. To investigate whether there is also a role for cardiac contractility in regulating Erbb2 downstream signalling pathways, we injected $T g(m y l 7: E G F P$ Has.HRAS); Tg(myl7:nrg2a-202-p2a-tdTomato) embryos with tnnt $2 a$ morpholinos and imaged them by confocal microscopy at $78 \mathrm{hpf}$. Notably, we observed that myocardial overexpression of $n r g 2 a$ resulted in cardiomyocyte multilayering even in the absence of contractility (Fig. 6m,n). However, overexpressing $n r g 2 a$ throughout the endothelium, including the endocardium, by using a stable $T g$ (fli1a:nrg2a-p2a-tdtomato) line did not cause a cardiomyocyte multilayering phenotype in tnnt $2 a$ morphants (Supplementary Fig. 10). By injecting low amount of myl7:nrg2ap2a-tdTomato plasmid DNA in tnnt $2 a$ morphants, we also observed that mosaic expression of $n r g 2 a$ recruits both $n r g 2 a$ expressing and non-expressing cardiomyocytes to form a multilayered wall suggesting that $n r g 2 a$ affects the behaviour of cardiomyocytes via both autocrine and paracrine signals (Supplementary Fig. 11). Furthermore, it has been recently reported that $\mathrm{N}$-cadherin relocalizes to the basal side of cardiomyocytes during trabeculation ${ }^{45}$. We observed that $n r g 2 a$ overexpression in non-contractile cardiomyocytes was sufficient to drive the relocalization of Cdh2-EGFP molecules to 

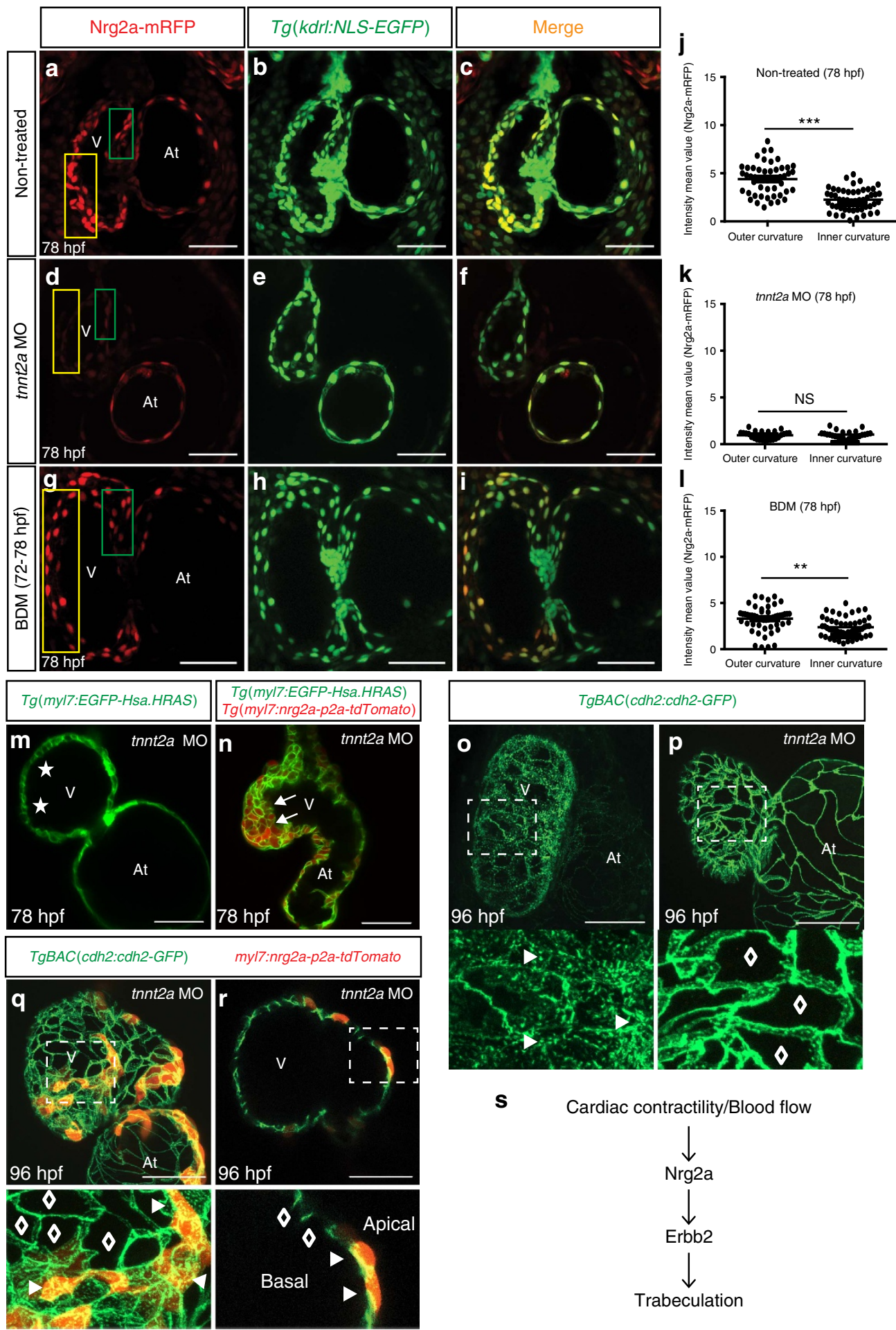

$\mathbf{S}$

Cardiac contractility/Blood flow

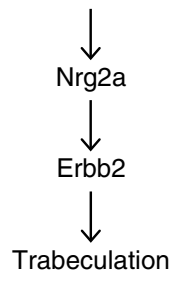

Figure 6 | Contractility/blood flow is required for endocardial expression of nrg2a but not the ability of cardiomyocytes to respond to $n r g 2 a$.

(a-i) Maximum intensity z-projections (25-30 z-stacks, mid-sagittal sections) of Tg(kdrl:NLS-EGFP);nrg2a+/- hearts from non-treated (a-c), tnnt2a MO injected (d-f) and BDM treated (g-i) $78 \mathrm{hpf} \mathrm{larvae;} \mathrm{scale} \mathrm{bars,} 50 \mu \mathrm{m}$. (j-I) Graphs showing cell-based Nrg2a-mRFP intensity in outer curvature (yellow box) and inner curvature (green box) of hearts from non-treated (j), tnnt2a MO (k) and BDM treated (I) 78 hpf larvae; dots represent individual Nrg2a-mRFP expressing endocardial cells. Values represent means \pm s.e.m.; ${ }^{\star \star} P \leq 0.01,{ }^{\star \star \star} P \leq 0.001$, NS (not significant), by Student's $t$-test. (m,n) $2 D$ confocal images (mid-sagittal sections) of 78 hpf $T g$ (myl7:EGFP-Hsa.HRAS) (m) or Tg(myl7:EGFP-Hsa.HRAS); Tg(myl7:nrg2a-p2a-tdTomato) hearts (n) showing that myocardial overexpression of nrg2a can induce cardiomyocyte multilayering in tnnt2a morphants; asterisks and arrows indicate single-layered and multilayered ventricular walls, respectively. (o-r) Maximum intensity z-projections of TgBAC(cdh2:cdh2-EGFP) hearts from non-injected (o), injected with tnnt2a MO alone (p) or injected with tnnt2a MO and myl7:nrg2a-p2a-tdTomato plasmid (q,r) 96 hpf larvae. (r) 2D confocal image (sagittal section) of heart shown in $\mathbf{q}$. Magnified images of dashed boxes are shown below (o-r); arrowheads and lozenges indicate presence and absence of Cdh2-EGFP proteins on the basal side of cardiomyocytes, respectively; At: atrium; $\vee$, ventricle; scale bars, $50 \mu \mathrm{m}$. (s) Schematic diagram of modulation of $\mathrm{Nrg} 2 \mathrm{a} /$ Erbb2 signaling by cardiac contractility/blood flow. 
their basal side (Fig. 6o-r, Supplementary Movie 1) while they remained mostly localized on the lateral sides of non- $n r g 2 a$ expressing cardiomyocytes, further indicating that cardiac contraction is not necessary for cardiomyocytes to respond to Nrg2a signalling. Altogether, these results support a model in which contractility/blood flow is required for endocardial expression of $n r g 2 a$ but not the expression or function of Nrg2a/Erbb2 signalling effectors (Fig. 6s).

\section{Discussion}

During trabeculation, cardiomyocytes undergo several morphogenetic changes required for the luminal growth and maintenance of the myocardial wall ${ }^{2,5,6,8,33,43,45}$. Taking advantage of high-resolution live imaging in the translucent zebrafish larvae, we investigated, using loss- and gain-of-function approaches, why trabeculation takes place primarily in the ventricle and how it is controlled by cardiac contractility/blood flow.

Given the importance of Nrgl in cardiac trabeculation in mouse ${ }^{11,46,47}$, we investigated the role of Nrg1 in zebrafish and found that it is not required for trabeculation, a finding consistent with a very recent publication ${ }^{48}$. Instead we found that Nrg2a is required for trabeculation in zebrafish while loss of Nrg2 does not lead to any obvious cardiac defects in mouse ${ }^{49}$. Nrg signalling plays many essential roles in vertebrate development and homeostasis $5,12,41,50,51$, and it will be interesting to investigate ligand selection across evolutionary time. We also observed that Nrg1 and Nrg2a signalling are not required for AV valve formation in zebrafish, consistent with previous observations of erbb2 mutants ${ }^{5}$. In mouse, Erbb2-Erbb3 heterodimer activity is required for early $\mathrm{AV}$ valve formation ${ }^{52,53}$, and it will be interesting to investigate the role of Erbb3 in zebrafish heart development.

To investigate why trabeculae form only in the ventricle, we used the $n r g 2 a^{m n 0237 G t /+}$ protein-trap line ${ }^{35}$ to monitor the endogenous expression of $n r g 2 a$, and found that it is expressed in the endocardium similar to mouse Nrg1 and Nrg2 (refs 54,55). Our results also indicate that Nrg2a-mRFP is strongly expressed in endocardial cells lining the outer curvature of the ventricle whereas it is weaker in other endocardial cells suggesting that some ventricular cardiomyocytes might be exposed to higher level of $\mathrm{Nrg} 2 \mathrm{a}$ signalling. One caveat here of course is that we examined the expression of a fusion protein which is likely to have very different stability than the endogenous Nrg2a protein.

Prior studies have also revealed the critical role of the cardiac jelly in endo-myocardial interactions ${ }^{16,56,57}$. Investigating the dynamics of cardiac jelly degradation during early cardiac development, we observed that by the time trabeculae appear, little cardiac jelly is left in the ventricle. By ectopic myocardial overexpression of $n r g 2 a$, we found that atrial cardiomyocytes could also respond to Nrg2a/Erbb2 signalling. We speculate that atrial cardiomyocytes receive less $\mathrm{Nrg}$ ligands, which are expressed by endocardial cells, due to the thickness of the cardiac jelly in the atrium and consequently do not form a multilayered wall.

When we overexpressed $n r g 2 a$ in cardiomyocytes using the myl7 promoter, we observed the formation of multiple continuous layers rather than the formation of trabeculae, possibly because of the high levels, even distribution and premature expression of $n r g 2 a$. We hypothesize that the expression of lower levels of $n r g 2 a$ in single cardiomyocytes starting at the right stage would lead them to delaminate and seed trabeculae as observed in wild-type embryos, and new transgenic tools will be required to test this hypothesis. In addition, higher levels of Nrg2a signalling might drive cardiomyocyte proliferation.

Several groups have shown the importance of physical forces in cardiac morphogenesis $6,24,27,58,59$. It has recently been reported that in zebrafish cardiac contraction is required for cardiac $n r g 1$ expression but not for cardiac erbb2 expression ${ }^{27}$. Here, we showed that contractility/blood flow is essential for cardiac expression of $n r g 2 a$. Interestingly, although $n r g 1$ upregulation by broad expression of a Notch intracellular domain was not sufficient to rescue trabeculation defects in tnnt $2 a$ morphants ${ }^{27}$, we could induce cardiomyocyte multilayering in these animals by myocardial overexpression of $n r g 2 a$. Moreover, we also showed that ectopic overexpression of $n r g 2 a$ in non-contractile cardiomyocytes led to the relocalization of Cdh2-EGFP to their basal side. Overall, these data indicate that contractility/blood flow is indispensable for cardiac expression of $n r g 2 a$ but not the ability of cardiomyocytes to respond to $n r g 2 a$.

We also observed that unlike myocardial specific $n r g 2 a$ overexpression, endocardial specific $n r g 2 a$ overexpression did not induce cardiomyocyte multilayering in tnnt $2 a$ morphants, likely due to the presence of a substantial gap between the endocardial and myocardial layers. These data further suggest that the thickness of the cardiac jelly may play an important role in determining when trabeculation starts.

In conclusion, our data indicate that trabeculae may initially form only in the ventricle in part because the cardiac jelly in the atrium does not get degraded as fast as it does in the ventricle. Atrial cardiomyocytes exhibit another kind of behaviour as they form the inner pectinate myofibers ${ }^{60,61}$, and it will be interesting to investigate the underlying signalling pathways. We also found that biomechanical forces work upstream of Nrg2a/Erbb2 signalling to modulate trabeculation in the zebrafish heart by modulating $n r g 2 a$ expression in the endocardium. It will be important to identify the proteins involved in sensing these forces as well as the transcriptional effectors regulating $n r g 2 a$ expression.

\section{Methods}

Zebrafish husbandry. Embryonic and adult zebrafish were raised and maintained under standard conditions ${ }^{62}$ and all animal experiments were done according to German Animal Protection Laws approved by the local governmental animal protection committee.

Zebrafish transgenic and mutant lines. In this study, we used the following transgenic and mutant lines: $\mathrm{Tg}\left(\mathrm{kdrl}\right.$ :Hsa.HRAS-mCherry) ${ }^{s 896}$ (ref. 39), $\mathrm{Tg}\left(\right.$ myl7:EGFP-Hsa.HRAS) ${ }^{s 883}$ (ref. 40), Tg(myl7:LIFEACT-GFP) ${ }^{s 974}$ (ref. 33), Tg(kdrl:NLS-EGFP)ubs1 (ref. 36), TgBAC(cdh2:cdh2-EGFP, crybb1:ECFP) ${ }^{z f 517}$ (ref. 63) abbreviated as TgBAC(cdh2:cdh2-EGFP), Tg(myl7:nrg2a202-p2atdTomato $)^{\text {bns } 140}$ abbreviated as Tg(myl7:nrg2a-p2a-tdTomato), Tg(fli1a:nrg2a202p2a-tdTomato $)^{b n s 199}$ abbreviated as Tg(fli1a:nrg2a-p2a-tdTomato) and $\mathrm{Tg}\left(\right.$ myl7:mVenus-gmnn) ${ }^{n c v 43 T g}$ (ref. 43) abbreviated as Tg(myl7:mVenus-gmnn), $n r g 2 a^{\text {mn0237Gt }}$ (ref. 35), erbb2 $2^{\text {st61 }}$ (ref. 64) and $n r g 1^{\text {bns101. }}$.

Generation of $\boldsymbol{n r g} \mathbf{1}^{\text {bns101 }}$ mutant. $n r g 1$ mutants were generated by TALEN mutagenesis targeting exon 2. The following TALEN arms were constructed and assembled using the Golden Gate method ${ }^{31}$.

TALEN arm1: NG NG NG NN NN HD NI NN NN NI NI NI HD HD HD NI NN TALEN arm2: HD NG NG NG NG HD HD NN NG NG HD NG NG NN NG NI HD HD

An amount of $100 \mathrm{pg}$ of total TALEN RNA and $50 \mathrm{pg}$ of GFP RNA (used to monitor injection efficiency) were coinjected into the cell at the one-cell stage.

The $n r g 1^{b n s 101}$ allele was genotyped using high-resolution melt analysis

(HRMA) with an Eco Real-Time PCR System (Illumina).

The following HRMA primers were used for PCR reactions

nrg1-exon2- F1 5'-GAGATTTTGGCAGGAAACCC-3'

nrg1-exon2- R1 5'-CTTTATGCTTTTGGGTTTG-3'.

Mosaic and stable overexpression of nrg2a. To generate a myocardial specific $n r g 2 a$ overexpressing construct, the $n r g 2 a-202$ coding sequence followed by p2a-tdTomato was cloned in a mini tol 2 plasmid harbouring a myl7 promoter using the Cold Fusion Cloning Kit (MC101A-1-SB). For mosaic expression of $n r g 2 a, 15$ pg of myl7:nrg2a-202-p2a-tdTomato plasmid DNA was coinjected with $10 \mathrm{ng}$ of Tol 2 RNA into the cell at the one-cell stage.

To establish the $\mathrm{Tg}(m y l 7: n r g 2 a 202-p 2 a-T o m a t o)^{b n s 140}$ line, injected larvae (F0) were screened for myocardial tdTomato fluorescence and raised to adulthood. 
After identifying founders, F1 embryos positive for fluorescence in their cardiomyocyte were raised to adulthood.

Morpholino injections. To stop cardiac contraction, $0.5 \mathrm{ng}$ of a tnnt $2 a$ morpholino ( $5^{\prime}$-CATGTTTGCTCTGATCTGACACGCA- $\left.3^{\prime}\right)$ was injected into one-cell stage embryos ${ }^{44}$.

Chemical treatments. To prevent contractility for $6 \mathrm{~h}$ (short term treatment), $72 \mathrm{hpf}$ zebrafish larvae were exposed to $20 \mathrm{mM} \mathrm{BDM}^{65}$. To block Erbb2 signalling, dechorionated embryos were treated with $10 \mu \mathrm{M}$ of the Erbb2 inhibitor PD 168393 (Calbiochem) from 36 to $96 \mathrm{hpf}$.

In vivo confocal imaging and data processing. Zebrafish embryos and larvae were mounted and anesthetized in $1.5 \%$ low-melt agarose (Sigma) containing 0.2 per cent tricaine on glass-bottom dishes. Before the gel solidified, the samples were manually oriented towards the microscope lens to enhance optical access to the heart. Images were captured with a Zeiss LSM780 or a spinning disk (CSU-X1 Yokogawa) confocal microscope using $\times 40(1.1 \mathrm{NA})$ and $\times 40(1.15 \mathrm{NA})$ water immersion lenses, respectively. The confocal data were then processed with the ZEN 2012 software (black edition). Nrg2a-mRFP intensity per cell was measured with the ZEN 2012 software (blue edition).

Whole mount in situ hybridization. To perform whole mount in situ hybridization $^{66}, 78 \mathrm{hpf}$ zebrafish larvae were fixed in $4 \%$ paraformaldehyde overnight at $4{ }^{\circ} \mathrm{C}$ and subsequently dehydrated in $100 \%$ methanol at $-20^{\circ} \mathrm{C}$. Larvae were rehydrated with $1 \times$ PBS and permeabilized by digestion with proteinase $\mathrm{K}$ $\left(10 \mu \mathrm{g} \mathrm{ml}^{-1}\right)$ at room temperature for $30 \mathrm{~min}$. After washing with $1 \times \mathrm{PBT}$ $(1 \times$ phosphate buffered saline (PBS), $0.1 \%$ Tween $20(\mathrm{vol} / \mathrm{vol}))$, larvae were hybridized with $200 \mathrm{ng}$ of $n r g 2 a$ antisense DIG-labelled RNA probe overnight at $70{ }^{\circ} \mathrm{C}$. The hybridized probes were then detected with alkaline phosphataseconjugated anti-Digoxigenin antibody (Roche, dilution 1:1,000) for three hours at room temperature and the signal was visualized with BM purple (Roche).

The probe for $n r g 2 a$ was amplified from 78 hpf heart cDNA using $n r g 2 a$ forward $5^{\prime}$-TCTGTGTGGCTCTTTTGGTG- $3^{\prime}$ and reverse $5^{\prime}$-GTGCGTC AGTCTGGTCTGTG-3' primers. The PCR fragment was subcloned into pGEM-T.

Statistical analysis. Data were processed with the Prism5 software. Values are presented as mean \pm s.e. $P$ values $\left({ }^{\star} P \leq 0.05,{ }^{*} P \leq 0.01,{ }^{* *} P \leq 0.001\right)$ were calculated using Student's $t$-test.

Data availability. The authors declare that all data supporting the findings of this study are available within the article and its Supplementary Information files or from the corresponding author upon reasonable request.

\section{References}

1. Staudt, D. \& Stainier, D. Uncovering the molecular and cellular mechanisms of heart development using the zebrafish. Annu. Rev. Genet. 46, 397-418 (2012).

2. Sedmera, D. \& Thomas, P. S. Trabeculation in the embryonic heart. Bioessays 18, 607-607 (1996).

3. Sedmera, D., Pexieder, T., Vuillemin, M., Thompson, R. P. \& Anderson, R. H. Developmental patterning of the myocardium. Anat. Rec. 258, 319-337 (2000).

4. Stankunas, K. et al. Endocardial Brgl represses ADAMTS1 to maintain the microenvironment for myocardial morphogenesis. Dev. Cell 14, 298-311 (2008).

5. Liu, J. et al. A dual role for ErbB2 signaling in cardiac trabeculation. Development 137, 3867-3875 (2010).

6. Staudt, D. W. et al. High-resolution imaging of cardiomyocyte behavior reveals two distinct steps in ventricular trabeculation. Development 141, 585-593 (2014).

7. Gassmann, M., Casagranda, F., Orioli, D. \& Simon, H. Aberrant neural and cardiac development in mice lacking the ErbB4 neuregulin receptor. Nature 378, 390 (1995).

8. Lee, K.-F. et al. Requirement for neuregulin receptor erbB2 in neural and cardiac development. Nature 378, 394-398 (1995).

9. Jenni, R., Rojas, J. \& Oechslin, E. Isolated noncompaction of the myocardium. N. Engl. J. Med. 340, 966-967 (1999).

10. Morris, J. K. et al. Rescue of the cardiac defect in ErbB2 mutant mice reveals essential roles of ErbB2 in peripheral nervous system development. Neuron 23, 273-283 (1999).

11. Crone, S. A. et al. ErbB2 is essential in the prevention of dilated cardiomyopathy. Nat. Med. 8, 459-465 (2002).

12. Lai, D. et al. Neuregulin 1 sustains the gene regulatory network in both trabecular and nontrabecular myocardium. Circ. Res. 107, 715-727 (2010)

13. Brutsaert, D. L. et al. The cardiac endothelium: functional morphology, development, and physiology. Prog. Cardiovasc. Dis. 39, 239-262 (1996).
14. Tian, Y. \& Morrisey, E. E. Importance of myocyte-nonmyocyte interactions in cardiac development and disease. Circ. Res. 110, 1023-1034 (2012).

15. Bowers, S. L. \& Baudino, T. A. Laying the groundwork for growth: cell-cell and cell-ECM interactions in cardiovascular development. Birth Defects Res. C Embryo Today 90, 1-7 (2010).

16. Ramasubramanian, A. et al. On the role of intrinsic and extrinsic forces in early cardiac S-looping. Dev. Dyn. 242, 801-816 (2013).

17. Suri, C. et al. Requisite role of angiopoietin-1, a ligand for the TIE2 receptor, during embryonic angiogenesis. Cell 87, 1171-1180 (1996).

18. Meyer, D. \& Birchmeier, C. Multiple essential functions of neuregulin in development. Nature 378, 386-390 (1995).

19. Bersell, K., Arab, S., Haring, B. \& Kühn, B. Neuregulin1/ErbB4 signaling induces cardiomyocyte proliferation and repair of heart injury. Cell 138, 257-270 (2009).

20. Polizzotti, B. D. et al. Neuregulin stimulation of cardiomyocyte regeneration in mice and human myocardium reveals a therapeutic window. Sci. Transl. Med 7, 281ra245-281ra245 (2015).

21. D'Uva, G. et al. ERBB2 triggers mammalian heart regeneration by promoting cardiomyocyte dedifferentiation and proliferation. Nat. Cell Biol. 17, 627-638 (2015).

22. Gemberling, M., Karra, R., Dickson, A. L. \& Poss, K. D. Nrg1 is an injuryinduced cardiomyocyte mitogen for the endogenous heart regeneration program in zebrafish. Elife 4, e05871 (2015).

23. Collins, M. M. \& Stainier, D. Y. Chapter twenty-five-organ function as a modulator of organ formation: lessons from zebrafish. Curr. Top. Dev. Biol. 117, 417-433 (2016).

24. Bartman, T. et al. Early myocardial function affects endocardial cushion development in zebrafish. PLoS Biol. 2, e129 (2004).

25. Auman, H. J. et al. Functional modulation of cardiac form through regionally confined cell shape changes. PLoS Biol. 5, e53 (2007).

26. Peshkovsky, C., Totong, R. \& Yelon, D. Dependence of cardiac trabeculation on neuregulin signaling and blood flow in zebrafish. Dev. Dyn. 240, 446-456 (2011).

27. Samsa, L. A. et al. Cardiac contraction activates endocardial Notch signaling to modulate chamber maturation in zebrafish. Development 142, 4080-4091 (2015).

28. Sedmera, D., Pexieder, T., Rychterova, V., Hu, N. \& Clark, E. B. Remodeling of chick embryonic ventricular myoarchitecture under experimentally changed loading conditions. Anat. Rec. 254, 238-252 (1999).

29. Berdougo, E., Coleman, H., Lee, D. H., Stainier, D. Y. \& Yelon, D. Mutation of weak atrium/atrial myosin heavy chain disrupts atrial function and influences ventricular morphogenesis in zebrafish. Development 130, 6121-6129 (2003).

30. Moorman, A., Webb, S., Brown, N. A., Lamers, W. \& Anderson, R. H. Development of the heart:(1) formation of the cardiac chambers and arterial trunks. Heart 89, 806-814 (2003).

31. Cermak, T. et al. Efficient design and assembly of custom TALEN and other TAL effector-based constructs for DNA targeting. Nucleic Acids Res. 39, 4942-4948 (2011).

32. Honjo, Y., Kniss, J. \& Eisen, J. S. Neuregulin-mediated ErbB3 signaling is required for formation of zebrafish dorsal root ganglion neurons. Development 135, 2615-2625 (2008).

33. Reischauer, S., Arnaout, R., Ramadass, R. \& Stainier, D. Y. Actin binding GFP allows $4 \mathrm{D}$ in vivo imaging of myofilament dynamics in the zebrafish heart and the identification of Erbb2 signaling as a remodeling factor of myofibril architecture. Circ. Res. 115, 845-856 (2014).

34. Clark, K. J. et al. In vivo protein trapping produces a functional expression codex of the vertebrate proteome. Nat. Methods 8, 506-512 (2011).

35. Westcot, S. E. et al. Protein-Trap insertional mutagenesis uncovers new genes involved in zebrafish skin development, including a Neuregulin 2a-based ErbB signaling pathway required during median fin fold morphogenesis. PLoS ONE 10, e0130688 (2015).

36. Blum, Y. et al. Complex cell rearrangements during intersegmental vessel sprouting and vessel fusion in the zebrafish embryo. Dev. Biol. 316, 312-322 (2008).

37. Lockhart, M., Wirrig, E., Phelps, A. \& Wessels, A. Extracellular matrix and heart development. Birth Defects Res. A Clin. Mol. Teratol. 91, 535-550 (2011).

38. Manasek, F. J. Histogenesis of the embryonic myocardium. Am. J. Cardiol. 25, 149-168 (1970).

39. Chi, N. C. et al. Foxn 4 directly regulates tbx $2 \mathrm{~b}$ expression and atrioventricular canal formation. Genes Dev. 22, 734-739 (2008).

40. D’Amico, L., Scott, I. C., Jungblut, B. \& Stainier, D. Y. A mutation in zebrafish hmgcrlb reveals a role for isoprenoids in vertebrate heart-tube formation. Curr. Biol. 17, 252-259 (2007).

41. Falls, D. L. Neuregulins: functions, forms, and signaling strategies. Exp. Cell Res. 284, 14-30 (2003).

42. Odiete, O., Hill, M. F. \& Sawyer, D. B. Neuregulin in cardiovascular development and disease. Circ. Res. 111, 1376-1385 (2012). 
43. Jiménez-Amilburu, V. et al. In vivo visualization of cardiomyocyte apicobasal polarity reveals epithelial to mesenchymal-like transition during cardiac trabeculation. Cell Rep. 17, 2687-2699 (2016).

44. Sehnert, A. J. et al. Cardiac troponin $\mathrm{T}$ is essential in sarcomere assembly and cardiac contractility. Nat. Genet. 31, 106-110 (2002).

45. Cherian, A. V., Fukuda, R., Augustine, S. M., Maischein, H.-M. \& Stainier, D. Y. $\mathrm{N}$-cadherin relocalization during cardiac trabeculation. Proc. Natl Acad. Sci. USA 113, 7569-7574 (2016).

46. Kramer, R. et al. Neuregulins with an Ig-like domain are essential for mouse myocardial and neuronal development. Proc. Natl Acad. Sci. USA 93, 4833-4838 (1996).

47. Wadugu, B. \& Kühn, B. The role of neuregulin/ErbB2/ErbB4 signaling in the heart with special focus on effects on cardiomyocyte proliferation. Am. J. Physiol. Heart Circ. Physiol. 302, H2139-H2147 (2012).

48. Samsa, L. A., Ito, C. E., Brown, D. R., Qian, L. \& Liu, J. IgG-containing isoforms of Neuregulin-1 are dispensable for cardiac trabeculation in zebrafish. PLoS ONE 11, e0166734 (2016).

49. Britto, J. M. et al. Generation and characterization of neuregulin-2-deficient mice. Mol. Cell. Biol. 24, 8221-8226 (2004).

50. Horie, T. et al. Acute doxorubicin cardiotoxicity is associated with miR-146ainduced inhibition of the neuregulin-ErbB pathway. Cardiovasc. Res. 87, 656-664 (2010).

51. Cantó, C. et al. Neuregulins increase mitochondrial oxidative capacity and insulin sensitivity in skeletal muscle cells. Diabetes 56, 2185-2193 (2007).

52. Camenisch, T. D., Schroeder, J. A., Bradley, J., Klewer, S. E. \& McDonald, J. A. Heart-valve mesenchyme formation is dependent on hyaluronan-augmented activation of ErbB2-ErbB3 receptors. Nat. Med. 8, 850-855 (2002).

53. Armstrong, E. J. \& Bischoff, J. Heart valve development endothelial cell signaling and differentiation. Circ. Res. 95, 459-470 (2004).

54. Gitler, A. D., Lu, M. M., Jiang, Y. Q., Epstein, J. A. \& Gruber, P. J. Molecular markers of cardiac endocardial cushion development. Dev. Dyn. 228, 643-650 (2003).

55. Carraway, III K. L. et al. Neuregulin-2, a new ligand of ErbB3/ErbB4-receptor tyrosine kinases. Nature 387, 512-516 (1997).

56. Nakamura, A. \& Manasek, F. J. An experimental study of the relation of cardiac jelly to the shape of the early chick embryonic heart. Development 65, 235-256 (1981).

57. Mellman, K., Huisken, J., Dinsmore, C., Hoppe, C. \& Stainier, D. Y. Fibrillin-2b regulates endocardial morphogenesis in zebrafish. Dev. Biol. 372, 111-119 (2012).

58. Hove, J. R. et al. Intracardiac fluid forces are an essential epigenetic factor for embryonic cardiogenesis. Nature 421, 172-177 (2003).

59. Dietrich, A.-C., Lombardo, V. A., Veerkamp, J., Priller, F. \& Abdelilah-Seyfried, S. Blood flow and Bmp signaling control endocardial chamber morphogenesis. Dev. Cell 30, 367-377 (2014).

60. Foglia, M. J., Cao, J., Tornini, V. A. \& Poss, K. D. Multicolor mapping of the cardiomyocyte proliferation dynamics that construct the atrium. Development 143, 1688-1696 (2016).

61. Singleman, C. \& Holtzman, N. G. Analysis of postembryonic heart development and maturation in the zebrafish, Danio rerio. Dev. Dyn. 241, 1993-2004 (2012).

62. Westerfield, M. in The Zebrafish Book (University of Oregon Press, 2000).
63. Revenu, C. et al. Quantitative cell polarity imaging defines leader-to-follower transitions during collective migration and the key role of microtubuledependent adherens junction formation. Development 141, 1282-1291 (2014)

64. Lyons, D. A. et al. erbb3 and erbb2 are essential for schwann cell migration and myelination in zebrafish. Curr. Biol. 15, 513-524 (2005).

65. Higuchi, H. \& Takemori, S. Butanedione monoxime suppresses contraction and ATPase activity of rabbit skeletal muscle. J. Biochem. 105, 638-643 (1989).

66. Thisse, C. \& Thisse, B. High-resolution in situ hybridization to whole-mount zebrafish embryos. Nat. Protoc. 3, 59-69 (2008).

\section{Acknowledgements}

We would like to acknowledge Matthias Hammerschmidt for pointing out the late larval lethality in $n r g 2 a$ mutants and thank him and Stephen Ekker, Stephanie Westcot and Julia Hatzold for generously providing $n r g 2 a^{m n 0237 G t}$ animals prior to publication. We thank Radhan Ramadass for confocal imaging and technical support, Mohamed El-Brolosy, Vanesa Jimenez-Amilburu, Jason Lai, Anoop Cherian, Ryuichi Fukuda and Parisa Ghanbari for sharing reagents and helpful discussions, Sabine Fischer and Sharon Meaney-Gardian for excellent assistance, as well as Jean-Francois Matuszek, Sophie Mucenieks, Rita Retzloff and Martin Laszczyk for zebrafish care. This work was supported by the Max Planck Society and the Leducq Foundation.

\section{Author contributions}

S.J.R. and D.Y.R.S. designed the experiments. S.J.R. performed all the experiments. S.J.R and D.Y.R.S. analysed data, wrote and revised the manuscript. D.Y.R.S. supervised the project.

\section{Additional information}

Supplementary Information accompanies this paper at http://www.nature.com/ naturecommunications

Competing interests: The authors declare no competing financial interests.

Reprints and permission information is available online at http://npg.nature.com/ reprintsandpermissions/

How to cite this article: Rasouli, S. J. et al. Regulation of cardiomyocyte behavior in zebrafish trabeculation by Neuregulin 2a signaling. Nat. Commun. 8, 15281 doi: 10.1038/ncomms15281 (2017).

Publisher's note: Springer Nature remains neutral with regard to jurisdictional claims in published maps and institutional affiliations.

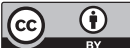

This work is licensed under a Creative Commons Attribution 4.0 International License. The images or other third party material in this article are included in the article's Creative Commons license, unless indicated otherwise in the credit line; if the material is not included under the Creative Commons license, users will need to obtain permission from the license holder to reproduce the material. To view a copy of this license, visit http://creativecommons.org/licenses/by/4.0/

(C) The Author(s) 2017 